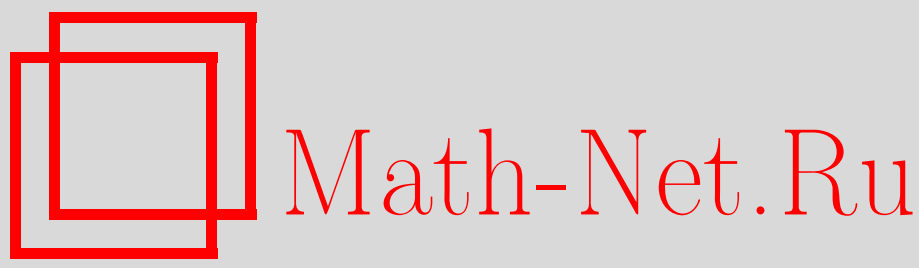

Д. В. Осин, О росте ранга подгрупп в конечно порожденных группах, Матем. сб., 1999, том 190, номер 8, 81-102

DOI: https://doi.org/10.4213/sm421

Использование Общероссийского математического портала Math-Net.Ru подразумевает, что вы прочитали и согласны с пользовательским соглашением

http: //www. mathnet.ru/rus/agreement

Параметры загрузки:

IP : 44.207 .124 .84

26 апреля 2023 г., 17:34:54 


\title{
О росте ранга подгрупп в конечно порожденных группах
}

\begin{abstract}
В работах [1] и [2] независимо введены и исследованы функции роста ранга для подгрупп конечно порожденной свободной группы. В предлагаемой статье понятие роста ранга распространяется на подгруппы произвольной конечно порожденной группы $G$ и исследуется зависимость асимптотического поведения введенных функций от выбора конечного порождающего множества в $G$. Для широкого класса групп (включающего, в частности, свободные полинильпотентные группы) получены оценки роста ранга подгруп, обобщающие известный результат Баумслага-Эйделькинда о конечно порожденных нормалњных делителях. Рассматриваются также некоторые вопросы, связанные с реализацией произволных функций в качестве функций роста ранга подгрупп в разрешимых группах.

Библиографоия: 12 названий.
\end{abstract}

\section{§1. Введение}

Хорошо известно, что подгруппа $H$ конечно порожденной группы $G$ не обязательно обладает конечной системой порождающих. В этом случае, используя словарную функцию длины на $G$, можно ввести некоторьй асимптотический инвариант - функцию роста ранга, который несет гораздо больше информации о подгруппе, нежели простая констатация отсутствия конечного порождающего множества.

Для подгрупп свободной группы конечного ранга такой инвариант независимо изучался в работах [1] и [2]. Напомним некоторые основные определения и результаты. Пусть $H$ - подгруппа свободной группы $F$ с конечньм базисом $X$; значения функиии роста ранга $r_{H}$ подгрупшы $H$ определяются следующим образом: $r_{H}(n)$ есть ранг подгруппы $H_{n}$, порожденной всеми элементами подгруппы $H$, длина которых относительно $X$ не превосходит $n$.

Ясно, что при малых значениях параметра $n$ поведение введенной функции сильно зависит от выбора системы порождающих в $F$. Поэтому изучение функций роста ранга проводится с точностью до некоторой эквивалентности, индуцированной отношением частичного порядка на множестве $\Omega$ всех функций $f: \mathbb{N} \rightarrow \mathbb{N}$. Более точно, пусть $f, g \in \Omega$; будем говорить, что $f$ асимптотически не превосходит $g$ и писать $f \preceq g$, если сушествуют такие константы $c, k \in \mathbb{N}$, что

$$
f(n) \leqslant k g(c n)
$$

для всех $n \in \mathbb{N}$. Отметим сразу, что для доказательства неравенства $f \preceq g$ достаточно проверить выполнение условия (1.1) для всех достаточно больших $n \in \mathbb{N}$. Далее, положим

$$
f(n) \sim g(n) \Longleftrightarrow f(n) \preceq g(n) \text { и } g(n) \preceq f(n) .
$$


Класс эквивалентности функции $f$ будем обозначать $[f]$.

В [1] и [2] показано, что класс эквивалентности функции роста ранга подгруппы не зависит от выбора конечной системы порождающих в $F$. Доказано также, что в случае, когда подгруппа $H$ нормальна в $F$, имеет место следуюшее обобшение формулы Шрайера:

$$
r_{H} \sim \gamma_{F / H},
$$

где $\gamma_{F / H}-$ функция роста факторгруппы $F / H$ (напомним, что функция роста конечно порожденной группы $K$ задается равенством $\gamma_{K}(n)=\operatorname{card}\{k \in K: l(k) \leqslant n\}$, где $l(k)$ - длина элемента $k$ относительно выбранной системы порождающих в $K)$. $\mathrm{C}$ другой стороны, оказывается, что любая неубываюшая функция $f \in \Omega$, не превосходящая некоторой экспоненты, может быть реализована с точностью до эквивалентности как функция роста ранга некоторой (не обязательно нормальной) подгрупшы.

В терминах введенных функций в [1] и [2] независимо сформулированно и доказано обобшение известной теоремы Хаусона (см. [3]): рост ранга пересечения двух подгрупп асимптотически не превосходит произведения ростов ранга этих подгрупп.

В настоящей статье понятие роста ранга переносится на случай подгрупп произвольных конечно порожденных групп. При этом оказывается, что непосредственное распространение определения приводит к функциям (названным функциями роста специального ранга), класс эквивалентности которых зависит от выбора конечного порождаюшего множества в основной группе. Этим объясняется необходимость введения функций роста общего ранга, которые являются асимптотическими инвариантами подг рупп и в случае свободной основной группы совпадают с функциями роста специального ранга и с функциями роста ранга из [1] и [2].

Предлагаемая статья состоит из пяти параграфов. В $\S 2$ даны необходимые определения и формулировки основных результатов. В $\S 3$ доказаны теоремы, описывающие зависимость асимптотического поведения функций роста общего и специального ранга от выбора конечного порождаюшего множества в основной группе. Рост общего ранга в группах некоторого класса $\mathscr{K}$, включающего, в частности, свободные полинильпотентные группы, исследуется в $\S 4$. Наконец, некоторые вопросы, связанные с реализацией произвольных функций в качестве функций роста ранга подгрупп, обсуждаются в $\S 5$.

Автор выражает признательность А. Ю. Ольшанскому за постановку задачи и постоянное внимание к данной работе.

\section{§2. Формулировка основных результатов}

Пусть $G$ - произвольная группа с конечным порождающим множеством $X, H$ подгруппа в $G$. Определим на $G$ функцию длины $l: G \rightarrow \mathbb{N} \cup\{0\}$ относительно $X$, полагая для всякого $g \in G$ значение $l(g)$ равным длине кратчайшего слова в алфавите $X \cup X^{-1}$, представляюшего элемент $g$ (значение $l(1)$ считаем равным 0 ). Введенная таким образом словарная функция длины задает на подгруппе $H$ фильтрацию

$$
\{1\}=H_{0} \subseteq H_{1} \subseteq H_{2} \subseteq \cdots,
$$


где $H_{n}$ - подгруппа, порожденная всеми элементами $h \in H$, длина которых не превосходит $n$, т.е.

$$
H_{n}=\operatorname{gp}\{h \in H: l(h) \leqslant n\} .
$$

ОПРЕДЕЛЕНИЕ 2.1. Наименьшее возможное число порождающих произволь-

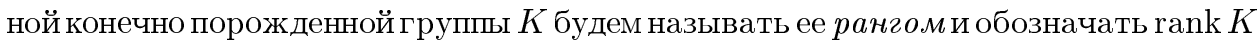
(для единичной группы $E$ считаем $\operatorname{rank} E=1$ ).

ОПРЕДЕЛЕНИЕ 2.2. Функции $r_{H}^{*}, r_{H}: \mathbb{N} \rightarrow \mathbb{N}$, заданные равенствами

$$
\begin{gathered}
r_{H}^{*}(n)=\operatorname{rank} H_{n}, \\
r_{H}(n)=\min _{H_{n} \subseteq K \subseteq H} \operatorname{rank} K
\end{gathered}
$$

(минимум берется по всевозможным подгруппам $K$, содержашим $H_{n}$ и содержащимся в $H$ ), будем называть соответственно функиией роста специального и общего ранга подгруппы $H$ относительно порождаюшего множества $X$.

Если основная $G$ группа свободна, то подгруппы $H_{n}$ являются свободными множителями в $H$ (см. [1]). Отсюда следует, что $\min _{H_{n} \subseteq K \subseteq H} \operatorname{rank} K=\operatorname{rank} H_{n}$ и $r_{H}^{*} \equiv r_{H}$. Принимая во внимание последнее равенство, для подгрупп свободных групп будем говорить просто о функииях роста ранга.

Оказывается, что функции роста специального ранга подгрупп могут, вообще говоря, меняться достаточно произвольным образом при замене конечного порождающего множества в основной групе. Легко заметить, что для любой функции $f \in \Omega$, реализуюшейся в качестве функции роста ранга (специального или обшего) некоторой подгруппы $H$ в конечно порожденной группе $G$, выполнено условие

(а) $f(n) \leqslant a^{n}$ для любых $n \in \mathbb{N}$ при некотором фиксированном $a$.

Если, кроме того, подгруппа $H$ не является локально циклической, то выполнено условие

(b) $f(n)>1$ при всех достаточно больших $n$.

Имеет место следуюшая

ТЕОРема 2.1. Для любъх двух функиий $f, g \in \Omega$, удовлетворяющих условиям (a) $u(\mathrm{~b})$, существуют такие группа $G$, подгруппа $H \subset G$ и две конечных системы порождающих $U$ u $V$ группь $G$, что при всех достаточно больших $n$

$$
r_{H}^{*(U)}(n)=f(n), \quad r_{H}^{*(V)}(n)=g(n)
$$

где $r_{H}^{*(U)}$ и $r_{H}^{*(V)}$ - функции роста специального ранга подгруппь $H$ относительно порожсдаюих множеств $U$ и $V$ соответственно.

Заметим, что выполнение условия (b) существенно, так как для локально циклической подгруппы имеем $r_{H}^{*} \equiv 1$ для любой системы порождающих в основной группе.

В противоположность теореме 2.1 будет доказана 
ТеОрема 2.2. Пусть $G$ - произвольная группа, $U$ и $V$ - два конечных порожсдающих множества в $G$ и $H-$ подгруппа в $G$. Тогда для любъх двух конечных порождающих множеств $U$ и $V$ группь $G$ имеем

$$
r_{H}^{(U)} \sim r_{H}^{(V)}
$$

Таким образом, рост общего ранга является асимптотическим инвариантом подгруппы и представляет по сравнению с ростом специального ранга значительно больший интерес. В дальнейшем, говоря о функции роста общего ранга, мы будем подразумевать соответствующий класс эквивалентности, не уточняя, относительно какого именно конечного порождающего множества в $G$ она вычисляется.

В качестве примера мы исследуем функции роста общего ранга в достаточно широком классе груп, включаюшем, в частности, свободные полинильпотентные групшы. Пусть, как обычно, $\gamma_{m} G$ обозначает $m$-й член нижнего центрального ряда групшы $G$ (т.е. $\left.\gamma_{1}(G)=G, \gamma_{j+1}(G)=\left[\gamma_{j}(G), G\right]\right)$. Напомним, что свободная полинильпотентная группа, отвечающая последовательности натуральных чисел $n_{1}, \ldots, n_{k},-$ это свободная группа в многообразии $\mathfrak{P}_{\left(n_{1}, \ldots, n_{k}\right)}$ всех групш $G$, удовлетворяюших тождеству $\gamma_{n_{k}}, \ldots, \gamma_{n_{1}} G=1$. Мы будем рассматривать групшы несколько более обшего вида $F / \gamma_{m} R$, где $F$ - свободная группа конечного ранга, $R$ - нетривиальная нормальная подгруппа в $F, m>1$ и факторгруппа $F / R$ разрешима; класс всех таких групп будем обозначать $\mathscr{K}$. Аналогом формулы (1.3) для групп класса $\mathscr{K}$ является следующее утверждение.

Tеорема 2.3. Пусть $G=F / \gamma_{m} R \in \mathscr{K}, A=R / \gamma_{m} R, \varepsilon-$ естественный гомоморфизм $G \rightarrow \bar{G}=F / R, H$ - нетривиальная нормальная подгруппа в $G$,

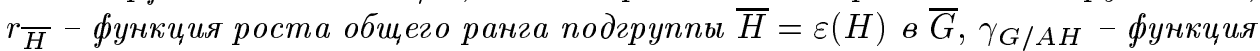
роста факторгруппь $G / A H$. Тогда

$$
\gamma_{G / A H} \preceq r_{H} \preceq r_{\bar{H}} \cdot\left(\gamma_{G / A H}\right)^{m-1}+\left(\gamma_{G / A H}\right)^{2 m-3} .
$$

В некоторых случаях функции роста общего ранга допускают более точные оценки.

СлеДСТВИЕ 2.1. Пусть $G=F /[R, R]$, причем группа $F / R$ полициклична. Пусть, кроме того, $H$ - нетривиальная нормальная подгруппа в $G$; обозначим $A=R /[R, R]$. Если при этом факторгруппа $G / A H$ содержсит нильпотентную подгруппу $L$ ступени $t$ конечного индекса, то

$$
r_{H} \sim n^{d}
$$

¿дe

$$
d=\sum_{i=1}^{t} i \cdot \operatorname{dim}_{\mathbb{Z}}\left(\gamma_{i} L / \gamma_{i+1} L\right),
$$

$u \operatorname{dim}_{\mathbb{Z}}\left(\gamma_{i} L / \gamma_{i+1} L\right)$ обозначает размерность абелевой әруппьи $\gamma_{i} L / \gamma_{i+1} L$ как $\mathbb{Z}$-модуля; в противном случае

$$
r_{H} \sim 2^{n}
$$


С помощью следствия 2.1 можно, в частности, вычислять функции роста общего ранга в свободных группах произведения многообразия абелевых групп на многообразие нильпотентных фиксированной ступени. Так, например, для коммутанта $G^{\prime}$ свободной метабелевой групшы $G$ ранга $s$ имеем:

$$
r_{G^{\prime}} \sim n^{s}
$$

Теорема 2.3 позволяет также перенести известньй результат Баумслага-Эйделькинда о конечно порожденных нормальных подгруппах свободной полинильпотентной группы (см. [4], [5]) на случай произвольных групп класса $\mathscr{K}$.

СлеДСТВИЕ 2.2. В группе $G=F / \gamma_{m} R \in \mathscr{K}$ нормальная подгруппа $H \neq 1$ тогда и только тогда имеет конечное число образующих, когда ее индекс конечен по модулю $A=R / \gamma_{m} R$, m.e. $|G / A H|<\infty$.

Последняя часть предлагаемой работы посвящена описанию множества функций $\Omega(\mathfrak{U})$, которые реализуются с точностью до эквивалентности в качестве функций роста общего ранга подгрупп (не обязательно нормальных) в конечно порожденных группах некоторых заданных многообразий $\mathfrak{U}$.

Приведем сначала два примера. Обозначим для краткости $\Omega_{0}$ множество всех неубывающих функций $f \in \Omega$ таких, что $f(n) \leqslant a^{n}$ для некоторой константы $a$. Ясно, что все функции, реализуемые как функции роста общего ранга, лежат в $\Omega_{0}$. В случае многообразия всех групп $\mathfrak{O}$ мы имеем

$$
\Omega(\mathfrak{O})=\Omega_{0},
$$

так как любая функция $f \in \Omega_{0}$ может быть реализована с точностью до эквивалентности как функция роста ранга некоторой подгруппы абсолютно свободной группы (см. [1]). С другой стороны, легко заметить, что для многообразия $\mathfrak{N}_{c}$ нильпотентных групп ступени $c$ множество $\Omega\left(\mathfrak{N}_{c}\right)$ состоит только из ограниченных функций. Следующие теоремы предоставляют нам еще несколько не столь очевидных примеров подобного рода.

ОПРЕДЕЛЕНИЕ 2.3. Будем говорить, что функция $f$ натурального аргумента полиномиальна, если для некоторых фиксированных $c, d \in \mathbb{N} f(n) \leqslant c n$ для всех $n \in \mathbb{N}$.

Во избежание недоразумений отметим, что полиномиальная функция не обязана быть полиномом. Так, например, полиномиальными в нашем смысле являются функции $\sin n, \ln n, n^{\pi}$ и т. д. Множество полиномиальных функций $f \in \Omega_{0}$ будем обозначать $\Omega_{p}$. В следующих теоремах $\mathfrak{S}_{l}$, как обычно, обозначает многообразие разрешимых групп ступени $l$.

ТЕОРемА 2.4. Для многообразия метабелевых групп имеет место равенство

$$
\Omega\left(\mathfrak{S}_{2}\right)=\Omega_{p}
$$

Кроме того, всякая полиномиальная функиия $f \in \Omega_{p}$ может быть реализована с точностью до әквивалентности в качестве функиии роста общего ранга некоторой подгруппь свободной метабелевой группь. 
При переходе от многообразия метабелевых групп к многообразиям $\mathfrak{S}_{l}$ при $l \geqslant 3$ ситуация сушественно меняется: оказывается, в этом случае

$$
\Omega\left(\mathfrak{S}_{l}\right)=\Omega_{0}
$$

Более того, имеет место

ТЕОРема 2.5. Любая функция $f \in \Omega_{0}$ может бить реализована с точностью до эквивалентности как функиия роста общего ранга некоторой подгруппь в свободной разрешимой группе ступени 3.

\section{§ 3. Асимптотическое поведение функций роста ранга}

Функции роста специального ранга. Предварительные результаты. При доказательстве теоремы 2.1 нам понадобится конструкция, использованная в работе [6]. Напомним основные понятия.

Пусть $F$ - свободная группа с базисом $X=\left\{x_{1}, \ldots, x_{s}\right\}, l_{F}: F \rightarrow \mathbb{N} \cup\{0\}-$ функция длины на $F$ относительно $X, T=\left\{t_{\lambda}\right\}$ - множество несократимых слов в алфавите $X^{ \pm 1}$ со следующими свойствами (для удобства изложения мы не делаем в дальнейшем различия между элементами свободных групп и представляющими их словами).

(i) Пусть $r$ - подслово некоторого слова $t_{\lambda} \in T$ и $l_{F}(r) \geqslant \frac{1}{50} l_{F}\left(t_{\lambda}\right)$. Тогда $r$ встречается в $t_{\lambda}$ лишь единожды.

(ii) Если, кроме того, $r$ является подсловом некоторого $t_{\mu} \in T^{ \pm 1}$, то $t_{\mu}=t_{\lambda}$.

Далее, пусть $w\left(t_{\lambda}\right)$ - некоторое слово в алфавите $T^{ \pm 1}$. Подставим в $w\left(t_{\lambda}\right)$ вместо элементов $t_{\lambda}$ множества $T$ их несократимые записи в порождающих $x_{1}, \ldots, x_{s}$ и проведем все возможные сокрашения. Полученное слово в алфавите $X^{ \pm 1}$ будем называть $T$-словом.

Предположим, что подгруппа $N \triangleleft F$ является нормальным замыканием в $F$ некоторого множества $T$-слов. Пусть также $G=F / N, \varepsilon: F \rightarrow G$ - естественный гомоморфизм. Нам понадобится следующая

Лемма 3.1. Пусть и - некоторое Т-слово. Тогда существует такое $T$-слово $v$, что $\varepsilon(v)=\varepsilon(u)$ и для всякого әлемента $w$ с условием $\varepsilon(w)=\varepsilon(u)$ выполнено неравенство $l_{F}(v) \leqslant l_{F}(w)$.

С точностью до обозначений утверждение леммы доказано в работе [6; лемма 8].

Лемма 3.2. Пусть $w$ - некоторое $T$-слово, представимое в $F$ в виде несократимого слова

$$
w=t_{\lambda_{1}}^{\alpha_{1}} \ldots t_{\lambda_{k}}^{\alpha_{k}}
$$

(все $\left.\alpha_{i}= \pm 1\right)$ в алфавите $T^{ \pm 1}$. Тогда

$$
l_{F}(w) \geqslant \max _{i=1, \ldots, k} l_{F}\left(t_{\lambda_{i}}\right)
$$


ДоКАЗАТЕЛЬСТВО проведем индукцией по $k$. При $k=1$ утверждение очевидно. Пусть $k=l+1$. Тогда $u=u_{0} t_{\lambda_{l+1}}^{\alpha_{l+1}}$, где $u_{0}=t_{\lambda_{1}}^{\alpha_{1}} \ldots t_{\lambda_{l}}^{\alpha_{l}}$ и длина $l_{F}\left(u_{0}\right) \geqslant$ $\max _{i=1, \ldots, l} l_{F}\left(t_{\lambda_{i}}\right)$. Ввиду условий (i) и (ii) возможные сокрашения на стыке $u_{0}$ и $t_{\lambda_{l+1}}^{\alpha_{l+1}}$ достаточно малы. Точнее, $l_{F}(u) \geqslant l_{F}\left(u_{0}\right)+l_{F}\left(t_{\lambda_{l+1}}\right)-c$, где $c \leqslant \frac{2}{50} l_{F}\left(t_{\lambda_{l+1}}\right)$ и $c \leqslant \frac{2}{50} l_{F}\left(t_{\lambda_{l}}\right)$. Последнее неравенство вместе с предположением индукции влечет $c \leqslant \frac{2}{50} l_{F}\left(u_{0}\right)$. Значит, $l_{F}(u)>\max \left\{l_{F}\left(u_{0}\right) ; l_{F}\left(t_{\lambda_{l+1}}\right)\right\}$. Теперь утверждение следует из предположения индукции.

Из доказанной леммы, в частности, вытекает, что всякая подгруппа в $F$, порожденная некоторьм подмножеством множества $T$, порождается им свободно.

Экспоненциальные множества слов. Как и в работе [6], нам потребуются вспомогательные множества слов с экспоненциальным ростом. Пусть $\mathscr{A}$ - множество слов в конечном алфавите $X, l: \mathscr{A} \rightarrow \mathbb{N}$ - функция длины относительно $X$.

ОПРЕДЕЛЕНИЕ 3.1. Сферической функиией роста множества $\mathscr{A}$ назовем функцию

$$
\sigma_{\mathscr{A}}(n)=\operatorname{card} \mathscr{A}_{n}
$$

где

$$
\mathscr{A}_{n}=\{a \in \mathscr{A}: l(a)=n\}
$$

Пусть $c \in \mathbb{N}$ - некоторая фиксированная константа (ее значение будет определено позднее). Положим $X^{k}=\left\{x_{k 1}, \ldots, x_{k c}\right\}, k=1, \ldots, 100$, и рассмотрим множество $\mathscr{A}^{k}$ всех слов в алфавите $X^{k}$. Легко заметить, что

$$
\sigma_{\mathscr{A}^{k}}(n)=c^{n}
$$

Занумеруем элементы множества $\mathscr{A}_{n}^{k}$ в произвольном порядке: $a_{n 1}^{(k)}, \ldots, a_{n, c^{n}}^{(k)}$ и рассмотрим множество $\mathscr{B}_{n}$ слов $b_{n i}$ в алфавите $X_{0}=\left(\bigcup_{k=1}^{100} X^{k}\right) \cup\{z, s\}$, заданных следуюшим образом. Пусть $n=100 m+k$, где $1 \leqslant k \leqslant 100$. Тогда положим

$$
b_{n i}=a_{m-1,100 i+1}^{(k)} z a_{m-1,100 i+2}^{(k)} z \ldots a_{m-1,100 i+100}^{(k)} z s^{k},
$$

где $i=0,1, \ldots,\left[c^{m-1} / 100\right]-1\left(\right.$ если $\left[c^{m-1} / 100\right]=0$, то полагаем $\left.\mathscr{B}_{n}=\varnothing\right)$. Легко проверить, что длина слова $b_{n i}$ относительно $X_{0}$ равна $n$. Далее, пусть

$$
\mathscr{B}=\bigcup_{n=1}^{\infty} \mathscr{B}_{n}
$$

Нам потребуются некоторые свойства построенного множества.

ЛЕмма 3.3. Для любых двух функиий $f, g \in \Omega$ не более, чем әкспоненциального роста, существует такая константа с, что для соответствующего множества $\mathscr{B}$ выполняется неравенство

$$
f(n)+g(n)<\sigma_{\mathscr{B}}(n-1)
$$

при всех достаточно больиих $n$. 
ДокаЗАТЕЛЬство. По условию существует такая константа $c_{0}>1$, что $f(n+1)+g(n+1) \leqslant c_{0}^{n}$. Положим $c=c_{0}^{200}, n>200 \log _{c}\left(200 c^{3}\right)$. Тогда

$$
\begin{aligned}
\sigma_{\mathscr{B}}(n) & =\operatorname{card} \mathscr{B}_{n} \geqslant\left[\frac{c^{\left[\frac{n}{100}\right]-2}}{100}\right]>\frac{1}{100 c^{3}} c^{\frac{n}{100}}-1 \geqslant \frac{1}{200 c^{3}} c^{\frac{n}{100}} \\
& =c^{\left(\frac{1}{100}-\frac{\log _{c}\left(200 c^{3}\right)}{n}\right) n}>c^{\left(\frac{1}{100}-\frac{1}{200}\right) n}=c_{0}^{n} \geqslant f(n+1)+g(n+1) .
\end{aligned}
$$

Лемма доказана.

Доказательство теоремы 2.1. Пусть заданы некоторые функции $f, g: \mathbb{N} \rightarrow \mathbb{N}$, удовлетворяющие условиям (а) и (b). Изменяя, если это необходимо, значения функций $f$ и $g$ в конечном числе точек, мы можем предположить, что $f(n)>1$ и $g(n)>1$ для всех $n \in \mathbb{N}$.

Рассмотрим последовательность свободных груп $F_{1}, G_{1}, F_{2}, G_{2}, \ldots$, где $\operatorname{rank} F_{i}=f(i), \operatorname{rank} G_{i}=g(i)$. Пусть $\varphi_{i}: F_{i} \rightarrow G_{i}, \psi_{i}: G_{i} \rightarrow F_{i+1}-$ некоторые изоморфные вложения.

Рассмотрим индуктивный предел $S$ построенной последовательности. По определению индуктивного предела сушествуют изоморфные вложения $\tau_{i}: F_{i} \rightarrow S$ и $\sigma_{i}: G_{i} \rightarrow S$, делающие коммутативной следующую диаграмму:

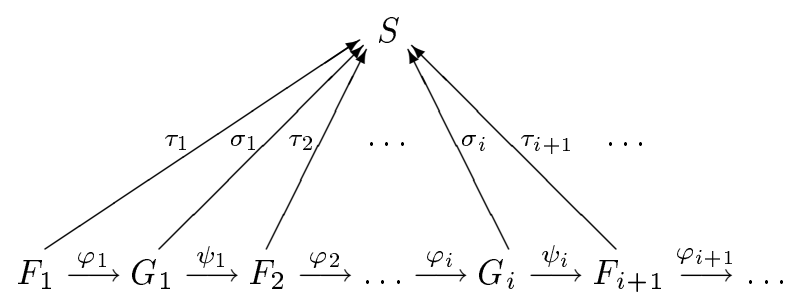

Обозначим через $f_{i 1}, \ldots, f_{i f(i)}$ и $g_{i 1}, \ldots, g_{i g(i)}$ свободные порождающие образов в $S$ групп $F_{i}$ и $G_{i}$ соответственно. По лемме 3.3 выберем для функций $f(n)$ и $g(n)$ такую константу $c \in \mathbb{N}$, что для соответствующего множества $\mathscr{B}$

$$
f(n)+g(n)<\sigma_{\mathscr{B}}(n-1)
$$

для всех $n>K$ при некотором фиксированном (достаточно большом) $K$. Ясно, что множество $\left\{f_{i 1}, \ldots, f_{i f(i)}, g_{i 1}, \ldots, g_{i g(i)}: i>K\right\}$ порождает групшу $S$. Далее, пусть $R$ - свободная группа с базисом $\left\{r_{i 1}, \ldots, r_{i f(i)}, t_{i 1}, \ldots, t_{i g(i)}: i>K\right\}, Q-$ ядро естественного гомоморфизма $\mu: R \rightarrow S$, переводящего $r_{i j}$ в $f_{i j}, t_{i j}$ в $g_{i j}$. Зададим мономорфизм $\beta$ группы $R$ в свободную группу с базисом $X=X_{0} \cup\{p, q\}$, полагая

$$
\begin{aligned}
& \beta\left(r_{n i}\right)=b_{n-1, i} p \\
& \beta\left(t_{n j}\right)=b_{n-1, j+f(n)} p q
\end{aligned}
$$

где $n>K, i=1, \ldots, f(n), j=1, \ldots, g(n)$; корректность такого определения следует из неравенства (3.2). Кроме того, пусть $\lambda$ - естественное вложение $Q \rightarrow R$, изоморфизм $\alpha: Q \rightarrow N$ - ограничение $\beta, \eta$ - гомоморфизм $F \rightarrow G$ с ядром 
$\operatorname{Ker} \eta=N^{F}$, а гомоморфизмы $\nu$ и $\gamma$ определяются (единственным образом) требованием коммутативности диаграммы:

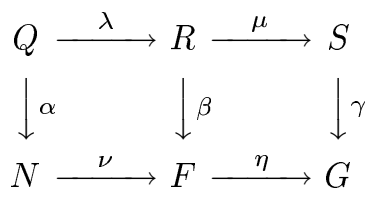

Положим

$$
T=\left\{b_{n-1, i} p, b_{n-1, j+f(n)} p q: n>K, i=1, \ldots, f(n), j=1, \ldots, g(n)\right\} .
$$

Покажем, что для множества $T$ выполнены условия (i) и (ii). Пусть $t \in T, r$ - подслово в $t$. Заметим, что из условия $l_{F}^{(X)}(r) \geqslant \frac{1}{50} l_{F}^{(X)}(t)$ следует, что $r$ содержит в качестве подслова слово вида $z a_{i j}^{(k)} z$. Действительно, предположив противное для слова $t=b_{n-1, j+f(n)} p q=a_{m-1,100 i+1}^{(k)} z a_{m-1,100 i+2}^{(k)} z \ldots a_{m-1,100 i+100}^{(k)} z s^{k} p q$, где $n-1=100 m+k, 1 \leqslant k \leqslant 100$, получим $l_{F}^{(X)}(r) \leqslant \max \{2+k+m ; 2(m-1)+1\}$. Легко проверить, что значения выражений $2+k+m$ и $2(m-1)+1$ при достаточно больших $m$ (без ограничения общности можно считать, что при всех $n>K$ ) меньше, чем $\frac{1}{50}(n+1)=\frac{1}{50} l_{F}^{(X)}(t)$. Аналогичньм образом мы приходим к противоречию в случае $t=b_{n-1, i}$. Остается заметить, что подслово $z a_{n j} z$ встречается в $t$ лишп единожды и не встречается ни в каком другом слове $t^{\prime} \in T^{ \pm 1}$.

Из выполнения условий (i) и (ii) для множества $T$ следует, что $\gamma$ является мономорфизмом (с точностью до обозначений это утверждение совпадает с леммой 6 работы [6]).

Пусть теперь $Y=X_{0} \cup\{p, p q\}, U=\eta(X), V=\eta(Y), l_{F}^{(X)}, l_{F}^{(Y)}-$ функции длины на $F$ относительно свободных порождаюших множеств $X$ и $Y, l_{G}^{(U)}, l_{G}^{(V)}$ - индуцированные функции длины на $G$ относительно $U$ и $V$ соответственно. Заметим, что

$$
\begin{array}{ll}
l_{F}^{(X)}\left(\beta\left(r_{i j}\right)\right)=i, & l_{F}^{(X)}\left(\beta\left(t_{i j}\right)\right)=i+1 \\
l_{F}^{(Y)}\left(\beta\left(r_{i j}\right)\right)=i, & l_{F}^{(Y)}\left(\beta\left(t_{i j}\right)\right)=i .
\end{array}
$$

Обозначим $H=\gamma(S)$ и положим

$$
\begin{aligned}
& H_{l}^{(U)}=\left\{h \in H: l_{G}^{(U)}(h) \leqslant l\right\}, \\
& H_{l}^{(V)}=\left\{h \in H: l_{G}^{(V)}(h) \leqslant l\right\} .
\end{aligned}
$$

Покажем, что при всех $l>K$

$$
\begin{aligned}
& H_{l}^{(U)}=\gamma \tau_{l}\left(F_{l}\right), \\
& H_{l}^{(V)}=\gamma \sigma_{l}\left(G_{l}\right) .
\end{aligned}
$$

Действительно, из формул (3.6), (3.7) и коммутативности диаграммы (3.5) следует, что $l_{G}^{(U)}\left(\gamma\left(f_{l j}\right)\right) \leqslant l$ при $l>K$, поэтому $\gamma \tau_{l}\left(F_{l}\right) \leqslant H_{l}^{(U)}$. 
Для доказательства включения $H_{l}^{(U)} \leqslant \gamma \tau_{l}\left(F_{l}\right)$ достаточно показать, что всякий элемент $h \in H$ длины $l_{G}^{(U)}(h) \leqslant l$ лежит в $\gamma \tau_{l}\left(F_{l}\right)$. Итак, пусть $h \in H$, $l_{G}^{(U)}(h) \leqslant l$ и $h_{0}-$ какой-нибудь прообраз элемента $h$ в группе $F$, представимый $T$-словом. По лемме 3.1 сушествует $T$-слово $w$, являющееся кратчайшим элементом в смежном классе $h=h_{0} N$. Но тогда по лемме $3.2 l_{F}^{(X)}(w)$ не меньше, чем длина любого $t_{\lambda_{i}} \in T$, входящего в запись (3.1). Следовательно, $w$ не содержит в своей записи элементов $t_{\lambda_{i}}$ длины большей, чем $l$. Значит, $w$ лежит в подгрупше $M$ группы $F$, порожденной теми из элементов $\beta\left(r_{i j}\right), \beta\left(t_{i j}\right)$, длина которых относительно $X$ не превосходит $l$. Но из коммутативности диаграммы $(3.5)$ и существования вложений $\varphi_{i}, \psi_{i}$ следует, что подгруппа $\eta(M) \leqslant H$ порождается образами $\eta \beta\left(r_{i j}\right), \eta \beta\left(t_{i j}\right)$ тех элементов $\beta\left(r_{i j}\right), \beta\left(t_{i j}\right)$, длина которых в точности равна $l$, т.е.

$$
\begin{aligned}
M & =\operatorname{gp}\left\{\eta \beta\left(r_{l 1}\right), \ldots, \eta \beta\left(r_{l f(l)}\right), \eta \beta\left(t_{l-1,1}\right), \ldots, \eta \beta\left(t_{l-1, g(l-1)}\right)\right\} \\
& =\operatorname{gp}\left\{\eta \beta\left(r_{l 1}\right), \ldots, \eta \beta\left(r_{l f(l)}\right)\right\}=\gamma \tau_{l}\left(F_{l}\right)
\end{aligned}
$$

(см. (3.6) и (3.7)). Так как элемент $h$ лежит в подгруппе $\eta(M)$, то включение $H_{l}^{(U)} \leqslant \gamma \tau_{l}\left(F_{l}\right)$, а с ним и равенство (3.8) доказаны. Доказательство равенства (3.9) аналогично. Следует лишь заменить всюду $X$ и $U$ соответственно на $Y$ и $V$, а вместо (3.10) написать

$$
\begin{aligned}
M & =\operatorname{gp}\left\{\eta \beta\left(r_{l 1}\right), \ldots, \eta \beta\left(r_{l f(l)}\right), \eta \beta\left(t_{l, 1}\right), \ldots, \eta \beta\left(t_{l, g(l)}\right)\right\} \\
& =\operatorname{gp}\left\{\eta \beta\left(t_{l 1}\right), \ldots, \eta \beta\left(t_{l g(l)}\right)\right\}=\gamma \sigma_{l}\left(G_{l}\right) .
\end{aligned}
$$

Остается заметить, что гомоморфизмы $\gamma \tau_{l}$ и $\gamma \sigma_{l}$ являются изоморфньми вложениями, а значит, группы $\gamma \tau_{l}\left(F_{l}\right)$ и $\gamma \sigma_{l}\left(G_{l}\right)$ свободны и $\operatorname{rank} \gamma \tau_{l}\left(F_{l}\right)=f(l)$, $\operatorname{rank} \gamma \sigma_{l}\left(G_{l}\right)=g(l)$. Из $(3.8)$ и (3.9) получаем

$$
\begin{aligned}
& r_{H}^{*(U)}(n)=f(n), \\
& r_{H}^{*(V)}(n)=g(n)
\end{aligned}
$$

при всех достаточно больших $n(n>K)$. Теорема доказана.

Функции роста общего ранга. Доказательство теоремы 2.2. Используя обозначения теоремы 2.2 , положим $U=\left\{u_{1}, \ldots, u_{s}\right\}, V=\left\{v_{1}, \ldots, v_{t}\right\}$. Каждый элемент множества $U$ представляется в виде некоторого слова в алфавите $V \cup V^{-1}$ : $u_{1}=w_{1}\left(v_{\lambda}\right), \ldots, u_{s}=w_{s}\left(v_{\lambda}\right)$. Положим

$$
c=\max _{i} l_{G}^{(V)}\left(w_{i}\right) .
$$

Покажем, что

$$
H_{n}^{(U)} \subseteq H_{c n}^{(V)},
$$

где $H_{n}^{(U)}$ и $H_{n}^{(V)}$ определены так же, как и в доказательстве теоремы 2.1. Пусть $h \in H$ и $l_{G}^{(U)}(h) \leqslant n$. Тогда, учитывая $(3.11)$, получим $l_{G}^{(V)}(h) \leqslant c n$, откуда следует вхождение (3.12). По определению функции роста общего ранга

$$
r_{H}^{(V)}(c n)=\min _{H_{c n}^{(V)} \subseteq K \subseteq H} \operatorname{rank} K \geqslant \min _{H_{n}^{(U)} \subseteq K \subseteq H} \operatorname{rank} K=r_{H}^{(U)}(n) .
$$

Аналогично доказывается, что для некоторого $k=\mathrm{const}$

$$
r_{H}^{(U)}(k n) \geqslant r_{H}^{(V)}(n) .
$$

Комбинируя (3.13) и (3.14), получаем утверждение теоремы 2.2. 
Функции роста ранга и гомоморфизмы. В настояшем пункте мы докажем одно вспомогательное утверждение, необходимое для дальнейшего изложения.

ЛЕмма 3.4. Пусть $G=\operatorname{gp}\left\{g_{1}, \ldots, g_{s}\right\}-$ произвольная группа, $H$ - подгруппа в $G, \varphi: G \rightarrow \bar{G}$ - эпимоморфизм и $\bar{H}=\varphi(H)$ - образ подгруппь $H$. Обозначим $r_{H}$ и $r_{\bar{H}}$ функции роста общего ранга подгрупп $H$ и $\bar{H}$ относительно порождающих множеств $\left\{g_{1}, \ldots, g_{s}\right\},\left\{\varphi\left(g_{1}\right), \ldots, \varphi\left(g_{s}\right)\right\}$ групп $G$ u $\bar{G}$ соответственно. Тогда

$$
\begin{aligned}
& r_{\bar{H}}^{*}(n) \leqslant r_{H}^{*}(n), \\
& r_{\bar{H}}(n) \leqslant r_{H}(n)
\end{aligned}
$$

для всех $n \in \mathbb{N}$.

ДокАЗАТЕЛЬство. Обозначим $\bar{l}$ функцию длины на $\bar{G}$ относительно системы порождающих $\left\{\varphi\left(g_{1}\right), \ldots, \varphi\left(g_{s}\right)\right\}, \bar{H}_{n}=\operatorname{gp}\{\bar{h} \in \bar{H}: \bar{l}(\bar{h}) \leqslant n\}$. Неравенство (3.15) следует из того, что $\bar{H}_{n}=\varphi\left(H_{n}\right)$. Далее, по определению $r_{H}(n)=$ $\min _{H_{n} \subseteq T \subseteq H} \operatorname{rank} T$. Пусть $r_{H}(n)=\operatorname{rank} T_{0}, H_{n} \subseteq T_{0} \subseteq H$. Таккак $\bar{H}_{n}=\varphi\left(H_{n}\right)$,

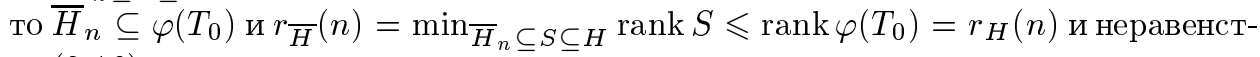
во (3.16) доказано.

В качестве следствия доказанной леммы получаем неравенство

$$
r_{\bar{H}} \preceq r_{H},
$$

где $r_{\bar{H}}$ и $r_{H}-$ функции роста общего ранга подгруппы $H$ и ее гомоморфного образа $\bar{H}$, вычисленные относительно произвольных конечных порождающих множеств в группах $G$ и $\bar{G}$. Действительно, в силу теоремы 2.2 неравенство (3.17) достаточно установить для каких-нибудь фиксированных конечных систем порождающих в $G$ и $\bar{G}$, что и сделано в лемме 3.4 .

\section{§4. Функции роста общего ранга подгрупп в группах класса $\mathscr{K}$}

Предварительные результаты. Прежде чем непосредственно перейти к доказательству теоремы 2.3, докажем некоторые вспомогательные результаты. При этом мы несколько раз воспользуемся очевидным при $r \geqslant 2$ числовым неравенстBOM

$$
\sum_{l=0}^{k} r^{l}<2 r^{k}
$$

В следующей лемме дается довольно грубая оценка ранга подгруппы в конечно порожденной нильпотентной группе, которая, однако, достаточна для наших целей. Более точная оценка может быть получена с помощью формулы Витта (cм. [7]).

ЛЕмма 4.1. Пусть $G-r$-порохсденная нильпотентная группа ступени $k$, $N-$ nодгруппа в $G$. Тогда

$$
\operatorname{rank} N<2 r^{k} .
$$


ДокАЗАТЕЛЬСТво. Пусть $1=G_{k} \triangleleft G_{k-1} \triangleleft \cdots \triangleleft G_{0}=G$ - нижний центральный ряд группы $G=\operatorname{gp}\left\{g_{1}, \ldots, g_{r}\right\}$. Хорошо известно, что факторгруппа $G_{i} / G_{i+1}$ порождается теми смежными классами, которые содержат простые $(i+1)$-кратные левонормированные коммутаторы $\left(g_{l_{1}}, \ldots, g_{l_{i+1}}\right)$ (см. [7]). Таким образом, по индукции легко установить, что $\operatorname{rank} G_{i} / G_{i+1} \leqslant r^{i+1}$. Обозначим $N_{i}=N \cap G_{i}$ и рассмотрим естественные гомоморфизмы $\varphi_{i}: G \rightarrow G / G_{i}$. Тогда $N_{i} / N_{i+1}=N_{i} /\left(N_{i} \cap G_{i+1}\right) \cong \varphi_{i+1}\left(N_{i}\right)$. Факторы $G_{i} / G_{i+1}$ абелевы, поэтому

$$
\operatorname{rank} N_{i} / N_{i+1}=\operatorname{rank} \varphi_{i+1}\left(N_{i}\right) \leqslant \operatorname{rank} G_{i} / G_{i+1} \leqslant r^{i+1} .
$$

Но группы $N_{i}$ образуют нормальный ряд в $N$, а значит,

$$
\operatorname{rank} N \leqslant \sum_{l=0}^{k-1} \operatorname{rank} N_{l} / N_{l+1} \leqslant \sum_{l=0}^{k-1} r^{l+1} .
$$

Теперь требуемое утверждение следует из (4.1) в случае $r \geqslant 2$ и является очевидньмм при $r=1$.

При доказательстве верхней оценки в неравенстве (2.1) решаюшую роль играет следуюшая

Лемма 4.2. Пусть группа $P=S T$ представляется в виде произведения своих нормальных подгрупп $S$ и $T$. Пусть, кроме того, $\operatorname{rank} P=r$, $\operatorname{rank} P / T=s$ u группа $T$ нильпотентна ступени $t$. Тогда

$$
\operatorname{rank} S \leqslant 2^{t+1} s r^{t}+2^{t+1} r^{2 t-1} .
$$

ДокаЗАТЕЛЬСТво. Пусть $\left\{x_{1}, \ldots, x_{r}\right\}$ и $\left\{\bar{y}_{1}, \ldots, \bar{y}_{s}\right\}$ - порождаюшие множества групп $P$ и $P / T$ соответственно, $\varphi: P \rightarrow P / T$ - естественный гомоморфизм. Рассмотрим множество $Y$, построенное следующим образом. Во-первых, включим в $Y$ произвольные прообразы $y_{1}, \ldots, y_{s}$ элементов $\bar{y}_{1}, \ldots, \bar{y}_{s}$, лежашие в подгрупше $S$. Далее, пусть элемент $\varphi\left(x_{i}\right)$ представляется в виде слова $w_{i}\left(\bar{y}_{\lambda}\right)$ в алфавите $\left\{\bar{y}_{1}^{ \pm 1}, \ldots, \bar{y}_{s}^{ \pm 1}\right\}$. Добавим к множеству $Y$ элементы вида $a_{i}=x_{i}^{-1} w_{i}\left(y_{\lambda}\right)$, $i=1, \ldots, r$, где $w_{i}\left(y_{\lambda}\right)-$ слово, полученное из $w_{i}\left(\bar{y}_{\lambda}\right)$ заменой $\bar{y}_{\lambda}$ на $y_{\lambda}$ и $\bar{y}_{\lambda}^{-1}$ на $y_{\lambda}^{-1}$. Ясно, что $a_{i} \in T$. Итак,

$$
Y=\left\{y_{1}, \ldots, y_{s}, a_{1}, \ldots, a_{r}\right\},
$$

где $y_{i} \in S, a_{i} \in T$. Легко проверить, что $Y$ тоже порождает $P$.

Факторгруппа $P / S$ является нильпотентной ступени не более $t$ и ранга не более $r$. Она может быть задана копредставлением

$$
P / S=\left\langle Y \mid r=1, r \in \mathfrak{R}_{1} \cup \mathfrak{R}_{2} \cup \mathfrak{R}_{3}\right\rangle,
$$

где $\mathfrak{R}_{1}$ - множество слов вида $y_{i}, i=1, \ldots, s, \mathfrak{R}_{2}$ - множество всех $(t+1)$-кратных коммутаторов элементов $a_{1}, \ldots, a_{r}$, а $\Re_{3}$ - множество слов, построенное следующим образом. Пусть $N$ - свободная нильпотентная группа ступени $t$ ранга $r$ со свободными порождающими $n_{1}, \ldots, n_{r}$, и $\tau$ - гомоморфизм $N \rightarrow P / S$, переводящий $n_{j}$ в $a_{j}$. Обозначим $K=\operatorname{Ker} \tau$. По лемме 4.1 ранг подгруппы $K$ меншше, чем $2 r^{t}$; 
пусть $k_{1}, \ldots, k_{q}\left(q<2 r^{t}\right)$ - некоторая система ее порождающих. Рассмотрим слова $v_{i}\left(n_{\lambda}\right)$, представляющие элементы $k_{i}$ в алфавите $\left\{n_{1}^{ \pm 1}, \ldots, n_{r}^{ \pm 1}\right\}$, и если $K \neq 1$, включим в $\mathfrak{R}_{3}$ слова вида $v_{i}\left(a_{\lambda}\right), i=1, \ldots, q$, полученные из $v_{i}\left(n_{\lambda}\right)$ заменой $n_{\lambda}$ на $a_{\lambda}$ и $n_{\lambda}^{-1}$ на $a_{\lambda}^{-1}$. Если же $K=1$, то положим $\mathfrak{R}_{3}=\varnothing$.

Покажем, что копредставление (4.2) действительно задает группу $P / S$. Ясно, что для всякого $r \in \mathfrak{R}_{1} \cup \mathfrak{R}_{2} \cup \mathfrak{R}_{3}$ равенство $r=1$ справедливо в группе $P / S$ (здесь и далее мы отождествляем слова $r \in \mathfrak{R}_{1} \cup \mathfrak{R}_{2} \cup \mathfrak{R}_{3}$ и представляемые ими элементы). Пусть теперь элемент $r$ представляется словом $u\left(y_{\lambda}, a_{\mu}\right)$ в алфавите $Y^{ \pm 1}$ и равен 1 в $P / S$. С помощью конечного числа вычеркиваний подслов вида $y_{\lambda}^{ \pm 1}$ мы можем редуцировать слово $u\left(y_{\lambda}, a_{\mu}\right)$ к слову $u_{0}\left(a_{\mu}\right)$, не содержащему $y_{\lambda}^{ \pm 1}$. Далее, элемент $n$ группы $N$, представляемый словом $u_{0}\left(n_{\mu}\right)$, получающимся из $u_{0}\left(a_{\mu}\right)$ соответствующей заменой, лежит в подгруппе $K$. Но тогда слово $u_{0}\left(n_{\mu}\right)$ (а значит, и $\left.u_{0}\left(a_{\mu}\right)\right)$ можно редуцировать к слову $u_{1}\left(n_{\mu}\right)$ (соответственно $\left.u_{1}\left(a_{\mu}\right)\right)$, представляюшему единичный элемент в групше $N$, с помошью конечного числа вычеркиваний и вставок подслов вида $v_{i}\left(n_{\lambda}\right)$ (соответственно $\left.v_{i}\left(a_{\lambda}\right)\right)$. Наконец, слово $u_{1}\left(a_{\mu}\right)$ редуцируется к пустому с помощью конечного числа вставок и вычеркиваний слов из $\mathfrak{R}_{2}$, так как соответствующее ему слово $u_{1}\left(n_{\mu}\right)$ редуцируется к пустому с помощью конечного числа вставок и вычеркиваний $(t+1)$-кратных коммутаторов элементов $n_{1}, \ldots, n_{r}$.

Далее, заметим, что равенство $r=1, r \in \mathfrak{R}_{2}$, выполняется в группе $P$ (так как $\left.a_{i} \in T\right)$, а значит,

$$
S=\left(\mathfrak{R}_{1} \cup \mathfrak{R}_{3}\right)^{P},
$$

т.е. подгруппа $S$ порождается элементами из $\mathfrak{R}_{1} \cup \mathfrak{R}_{3}$ как нормальньй делитель. Элементы $\mathfrak{R}_{3}$ будем кратко обозначать $v_{1}, \ldots, v_{q}$. Рассмотрим множество

$$
Z=\left\{y_{i},\left(y_{i}, a_{k_{1}}^{\varepsilon_{1}}\right), \ldots,\left(y_{i}, a_{k_{1}}^{\varepsilon_{1}}, \ldots, a_{k_{t}}^{\varepsilon_{t}}\right), v_{j},\left(v_{j}, a_{k_{1}}^{\varepsilon_{1}}\right), \ldots,\left(v_{j}, a_{k_{1}}^{\varepsilon_{1}}, \ldots, a_{k_{t-1}}^{\varepsilon_{t-1}}\right)\right\},
$$

где $\left(y_{i}, a_{k_{1}}^{ \pm 1}\right), \ldots,\left(y_{i}, a_{k_{1}}^{ \pm 1}, \ldots, a_{k_{t}}^{ \pm 1}\right),\left(v_{j}, a_{k_{1}}^{\varepsilon_{1}}\right), \ldots,\left(v_{j}, a_{k_{1}}^{\varepsilon_{1}}, \ldots, a_{k_{t-1}}^{\varepsilon_{t-1}}\right)$ - соответствуюшие левонормированные коммутаторы, $i=1, \ldots, s, j=1, \ldots, q, k_{l}=1, \ldots, r$, $\varepsilon_{l}= \pm 1, l=1, \ldots, t$. Покажем, что $S=$ gр $Z$. Для этого достаточно показать, что $z^{y} \in \operatorname{gp} Z$ для любых $z \in Z$ и $y \in Y^{ \pm 1}$. Рассмотрим возможные случаи.

1. $y=y_{i}^{ \pm 1}$. Очевидно, $z^{y_{i}^{ \pm 1}} \in \operatorname{gp} Z$ для любого $z \in Z$, так как $y_{i} \in Z$.

2. $y=a_{k}^{ \pm 1}$. Рассмотрим различные возможности для $z$.

а) $z=y_{i}$; тогда $z^{y}=y_{i}^{a_{k}^{ \pm 1}}=y_{i}\left(y_{i}, a_{k}^{ \pm 1}\right) \in \operatorname{gp} Z$.

б) $z=\left(y_{i}, a_{k_{1}}^{\varepsilon_{1}}, \ldots, a_{k_{l}}^{\varepsilon_{l}}\right), 1 \leqslant l<q$; тогда для сопряженного элемента получаем $z^{y}=\left(y_{i}, a_{k_{1}}^{\varepsilon_{1}}, \ldots, a_{k_{l}}^{\varepsilon_{l}}\right)\left(y_{i}, a_{k_{1}}^{\varepsilon_{1}}, \ldots, a_{k_{l}}^{\varepsilon_{l}}, a_{k}^{ \pm 1}\right) \in \operatorname{gp} Z$.

в) $z=\left(y_{i}, a_{k_{1}}^{\varepsilon_{1}}, \ldots, a_{k_{t}}^{\varepsilon_{t}}\right)$; тогда $z^{y}=z$, так как элемент $z$ лежит в центре групшы $T$.

Случаи $z=v_{j}, z=\left(v_{j}, a_{k_{1}}^{\varepsilon_{1}}, \ldots, a_{j_{l}}^{\varepsilon_{l}}\right), 1 \leqslant l<t-1$, и $z=\left(v_{j}, a_{k_{1}}^{\varepsilon_{1}}, \ldots, a_{k_{t-1}}^{\varepsilon_{t-1}}\right)$ рассматриваются аналогично.

Для доказательства леммы осталось оценить число элементов множества $Z$. Заметим, что $\operatorname{card} Z=s+s \cdot 2 r+\cdots+s \cdot(2 r)^{t}+q+q \cdot 2 r+\cdots+q \cdot(2 r)^{t-1}$. Учитывая, что $q<2 r^{t}$, окончательно получаем

$$
\operatorname{card} Z<s \cdot 2(2 r)^{t}+q \cdot 2(2 r)^{t-1}<2^{t+1} s r^{t}+2^{t+1} r^{2 t-1} .
$$


Вербальные сплетения. При доказательстве нижней оценки в неравенстве (2.1) нами будет использоваться вложение Шмелькина группы вида $F / V(R)$ в вербальное сплетение. Напомним основные определения (подробнее см. [8], [9]).

Пусть $\mathfrak{V}$ - произвольное многообразие, $V$ - множество слов в некотором алфавите, задаюшее тождества многообразия $\mathfrak{V}$. Для произвольной группы $G$ через $V(G)$ будем обозначать соответствующую вербальную подгруппу. Вербальным произведением $\mathfrak{V} \prod_{\lambda \in \Lambda} A_{\lambda}$ семейства груп $\left\{A_{\lambda}\right\}_{\lambda \in \Lambda}$, ассоциированным с многообразием $\mathfrak{V}$, назьвается факторгруппа свободного произведения $A=\prod_{\lambda \in \Lambda}^{*} A_{\lambda}$ по подгруппе $V(A) \cap\left[A_{\lambda}\right]^{*}$, где $\left[A_{\lambda}\right]^{*}$ - декартова подгруппа свободного произведения. Далее, пусть заданы две групшы $A$ и $B$. Дискретным вербальным сплетением $A$ wr $_{V} B$ групп $A$ и $B$, ассоциированным с многообразием $\mathfrak{V}$, называется расщепляемое расширение вербального произведения $\mathfrak{V} \prod_{b \in B} A(b)$ (базы сплетения) груп $A(b) \cong A$ с помошью группы $B$. При этом $b^{-1} a\left(b_{0}\right) b=a\left(b_{0} b\right)$, где $a(b)$ обозначает элемент группы $A(b)$, соответствующий элементу $a \in A$ при изоморфизме $A$ и $A(b)$.

Лемма 4.3 [9]. Пусть $F$ - абсолютно свободная группа со свободными образуюшими $x_{\mu}, \mu \in \mathrm{M}, u R \triangleleft F$. Обозначим через $U$ свободную группу в многообразии, соответствующем множсеству слов $V$, свободно порохсденную множсеством $u_{\mu}, \mu \in \mathrm{M}$. Тогда отобрахсение $x_{\mu} \rightarrow \bar{x}_{\mu} u_{\mu}(1)$, где $\bar{x}_{\mu}=x_{\mu} R \in F / R$, продолжсается до изоморфизма группь $F / V(R)$ и некоторой подгруппь вербального сплетения $U \operatorname{wr}_{V} F / R$.

Пусть теперь $G$ - группа вида $F / \gamma_{m} R$, где $F$ - свободная группа конечного ранга $r, m>1, R$ - нетривиальный нормальный делитель в $F$. По лемме 4.3 группа $G$ вложима в вербальное сплетение $S=U \mathrm{wr}_{V} \bar{G}$, где $V$ - множество тождеств многообразия нильпотентных групп ступени $m, U$ - свободная нильпотентная группа ранга $r$ и ступени $m, \bar{G}=F / R$. Пусть $\theta: G \rightarrow S$ - соответствующее вложение, $A=R / \gamma_{m} R$.

Обозначим через $T$ базу сплетения. Ясно, что $T$ является свободной нильпотентной группой ступени $m$ со свободным порождающим множеством $u_{1}(h), \ldots, u_{r}(h)$, где $h$ пробегает всю группу $\bar{G}$. Всякому неединичному элементу $t \in T$ поставим в соответствие семейство $\mathscr{P}_{t}$ всех таких множеств $X_{\lambda}=\left\{h_{1}, \ldots, h_{k}\right\}$, что $t$ представляется в виде

$$
t=u_{i_{1}}^{\alpha_{1}}\left(h_{1}\right) \ldots u_{i_{k}}^{\alpha_{k}}\left(h_{k}\right)
$$

Упорядочим множества $X_{\lambda}$, положив

$$
X_{\lambda} \leqslant X_{\mu} \Longleftrightarrow X_{\lambda} \subseteq X_{\mu}
$$

Покажем, что для всякого неединичного $t \in T$ семейство $\mathscr{P}_{t}$ содержит единственный минимальньй элемент. Действительно, пусть $X_{\lambda}$ и $X_{\mu}$ - два минимальных элемента некоторого семейства $\mathscr{P}_{t}$. Рассмотрим гомоморфизм $\varphi$ группы $T$ на свою подгруппу $\widehat{T}=\operatorname{gp}\left\{u_{1}(h), \ldots, u_{r}(h): h \in \bar{G} \backslash X_{\mu}\right\}$ с ядром $\operatorname{Ker} \varphi=$ $\operatorname{gp}\left\{u_{1}(h), \ldots, u_{r}(h): h \in X_{\mu}\right\}$. Ясно, что $\varphi(t)=1$. Но тогда $X_{\lambda} \subseteq X_{\mu}$, так как иначе, представляя элемент $t$ в виде (4.4) при $h_{1}, \ldots, h_{k} \in X_{\lambda}$ и учитьвая минимальность $X_{\lambda}$, получим $\varphi(t) \neq 1$. Аналогично доказывается, что $X_{\mu} \subseteq X_{\lambda}$. Значит, $X_{\lambda}=X_{\mu}$. 
ОПРЕДЕЛЕНИЕ 4.1. Пусть $g$ - произвольный элемент групшы $G$. Тогда его образ $\theta(a)$ единственным образом представим в виде $\bar{g} t$, где $\bar{g} \in \bar{G}, t \in T$. Минимальньй элемент семейства $\mathscr{P}_{t}$ назовем носителем элемента $g$ и обозначим supp $g$.

ЛЕмма 4.4. Пусть $a \in \gamma_{m-1} A, g-$ произвольный әлемент $G, \bar{g}$ - его образ при естественном гомоморфизме $\varepsilon: G \rightarrow \bar{G}$. Тогда

$$
\operatorname{supp} a^{g}=\left\{h \in \bar{G}: \bar{h} g^{-1} \in \operatorname{supp} a\right\}
$$

ДоказАТельство. Ясно, что $\theta(a) \in T$ и $\theta\left(a^{g}\right) \in T$. Кроме того, легко заметить, что из $a \in \gamma_{m-1} A$ следует, что $a^{g_{1}}=a^{g_{2}}$, если $\varepsilon\left(g_{1}\right)=\varepsilon\left(g_{2}\right)$. Проводя непосредственные вычисления, можно показать, что $\left\{h \in \bar{G}: \bar{h} g^{-1} \in \operatorname{supp} a\right\} \in \mathscr{P}_{a^{g}}$. Минимальность множества $\left\{h \in \bar{G}: \bar{g}^{-1} h \in \operatorname{supp} a\right\}$ следует из обратимости операции сопряжения.

Пусть $\bar{l}: \bar{G} \rightarrow \mathbb{N} \cup\{0\}$ - словарная функция длины на группе $\bar{G}$ относительно порождающего множества, полученного естественным путем из базиса групшы $F$. Обозначим $\rho: \bar{G} \times \bar{G} \rightarrow \mathbb{N} \cup\{0\}$ индуцированную правоинвариантную метрику, заданную равенством $\rho\left(\bar{g}_{1}, \bar{g}_{2}\right)=\bar{l}\left(\bar{g}_{1} \bar{g}_{2}^{-1}\right)$.

ОПРЕДЕЛЕНИЕ 4.2. Шириной элемента $a \in A$ назовем величину

$$
d(a)=\max _{h \in \operatorname{supp} a} \bar{l}(h) .
$$

Лемма 4.5. Пусть $a \in A, g_{1}, g_{2} \in G, \bar{g}_{1}, \bar{g}_{2}$ - образы элементов $g_{1}, g_{2}$ в zpynne $\bar{G}$. Ecлu $\rho\left(\bar{g}_{1}, \bar{g}_{2}\right)>2 d(a)$, mo $\operatorname{supp} a^{g_{1}} \cap \operatorname{supp} a^{g_{2}}=\varnothing$.

ДокАЗАТЕЛьство. Пусть $s \in \operatorname{supp} a^{g_{1}} \cap \operatorname{supp} a^{g_{2}}$. Из равенства (4.5) следует, что существуют такие элементы $s_{1}, s_{2} \in \operatorname{supp} a$, что $s=\bar{s}_{1} g_{1}=\bar{s}_{2} g_{2}$. Но тогда

$$
\rho\left(\bar{g}_{1}, \bar{g}_{2}\right)=\bar{l}\left(\bar{g}_{1} \bar{g}_{2}^{-1}\right)=l\left(\bar{g}_{1} s^{-1} s \bar{g}_{2}^{-1}\right)=l\left(s_{1}^{-1} s_{2}\right) \leqslant \bar{l}\left(s_{1}\right)+\bar{l}\left(s_{2}\right) \leqslant 2 d(a),
$$

что противоречит условию.

ЛЕмма 4.6. Пусть $a_{1}, \ldots, a_{l}$ - произвольные нетривиальные әлементь подгруппь $\gamma_{m-1} A$, причем носители $\operatorname{supp} a_{1}, \ldots, \operatorname{supp} a_{l}$ попарно не пересекаются. Тогда әлементы $a_{1}, \ldots, a_{l}$ не содержатся ни в какой подгруппе $D \leqslant \gamma_{m-1} A$ ранга, меньшего $l$.

ДокАзАтЕльство. Так как группа $\gamma_{m-1} A$ абелева, то достаточно показать, что элементы $a_{1}, \ldots, a_{l}$ линейно независимы. Последнее равносильно линейной независимости образов $\theta\left(a_{1}\right), \ldots, \theta\left(a_{l}\right) \in T$. Пусть

$$
\left(\theta\left(a_{1}\right)\right)^{\alpha_{1}} \ldots\left(\theta\left(a_{l}\right)\right)^{\alpha_{l}}=1,
$$

причем $\alpha_{i} \neq 0$ для некоторого $i$. Гомоморфизм $\varphi$ группы $T$ на свою подгруппу $\widehat{T}=\operatorname{gp}\left\{u_{1}(h), \ldots, u_{r}(h): h \in \operatorname{supp} a_{i}\right\}$ с ядром $\operatorname{Ker} \varphi=\operatorname{gp}\left\{u_{1}(h), \ldots, u_{r}(h):\right.$ $\left.h \in \bar{G} \backslash \operatorname{supp} a_{i}\right\}$ оставляет элемент $\left(\theta\left(a_{i}\right)\right)^{\alpha_{i}}$ неподвижным. С другой стороны, применяя $\varphi$ к обеим частям $(4.6)$, получим $\varphi\left(\theta\left(a_{i}\right)^{\alpha_{i}}\right)=1$ (так как при $j \neq i$ носители $\operatorname{supp} a_{j}$ лежат в $\bar{G} \backslash \operatorname{supp} a_{i}$, а значит, $\left.\theta\left(a_{j}\right) \in \operatorname{Ker} \varphi\right)$. Мы пришли к противоречию с условием $\alpha_{i} \neq 0$.

В заключение докажем частный случай нижней оценки функции роста общего ранга в неравенстве (2.1) (в дальнейшем общий случай будет сведен к доказанномy). 
ЛЕмма 4.7. Пусть $G$ - конечно порохсденная группа вида $F / \gamma_{m} R, m>1$, $A=R / \gamma_{m} R$ и $H \triangleleft G-$ произвольная нормальная подгруппа, лежащая в $\gamma_{m-1} A$. Тогда

$$
\gamma_{\bar{G}} \preceq r_{H}
$$

əде $\bar{G}=F / R$.

ДокАЗАТЕльство. Возьмем какой-нибудь элемент $a \in H$ и обозначим через $d$ ширину элемента $a$. Положим $\bar{G}_{n}=\{\bar{g} \in \bar{G}: \bar{l}(\bar{g}) \leqslant n\}$, где $\bar{l}$ по-прежнему обозначает словарную функцию длины на $\bar{G}$ относительно множества порождающих, являюшихся образами свободных порождаюших $F$. Рассмотрим семейство $\mathscr{B}$ всех шаров $B_{\bar{g}_{0}}(d)=\left\{\bar{g} \in \bar{G}: \rho\left(\bar{g}_{0}, \bar{g}\right) \leqslant d\right\}$ радиуса $d$ с центрами в элементах $\bar{g}_{0} \in \bar{G}_{n}$. Выберем в $\mathscr{B}$ максимальное подсемейство $\mathscr{B}_{0}$ попарно непересекаюшихся шаров. Любое такое подсемейство имеет мощность

$$
\operatorname{card} \mathscr{B}_{0} \geqslant \frac{\operatorname{card} \bar{G}_{n}}{\operatorname{card} B_{1}(2 d)},
$$

так как семейство шаров радиуса $2 d$ с центрами в тех же элементах покрывает все множество $\bar{G}_{n}$. Действительно, для всякого $\bar{g} \in \bar{G}_{n}$ найдется элемент $\bar{g}_{0}$, являющийся центром некоторого шара из $\mathscr{B}_{0}$, такой, что $\rho\left(\bar{g}_{0}, \bar{g}\right) \leqslant 2 d$ (иначе мы могли бы добавить к $\mathscr{B}_{0}$ еше один шар с центром в $\left.\bar{g}\right)$. Заметим, что

$$
\frac{\operatorname{card} \bar{G}_{n}}{\operatorname{card} B_{1}(2 d)}=c_{0} \gamma_{\bar{G}}(n)
$$

где $c_{0}=\left(\operatorname{card} B_{1}(2 d)\right)^{-1}$.

Далее, пусть $\bar{g}_{1}, \ldots, \bar{g}_{l}$ - центры шаров из подсемейства $\mathscr{B}_{0}, g_{1}, \ldots, g_{l}$ - их прообразы в группе $G$, кратчайшие относительно системы порождающих, получающейся естественным образом из базиса групшы $F$ (соответствующую функцию длины будем обозначать $l$ ). Рассмотрим набор элементов $h_{1}=a^{g_{1}}, \ldots, h_{l}=a^{g_{l}}$. Ясно, что $\rho\left(\bar{g}_{i}, \bar{g}_{j}\right)>2 d$ для любых $i, j=1, \ldots, l$. Значит, по лемме 4.5 носители $\operatorname{supp} h_{1}, \ldots, \operatorname{supp} h_{l}$ попарно не пересекаются. Но тогда из леммы 4.6 следует, что ранг любой подгруппы, содержащей $h_{1}, \ldots, h_{l}$, не менее $l$. Покажем, что при достаточно больших $n \in \mathbb{N} h_{1}, \ldots, h_{l} \in H_{3 n}$. Действительно, $l\left(h_{i}\right) \leqslant l(a)+2 l\left(g_{i}\right)$. Но при достаточно больших $n$ имеем $l(a) \leqslant n$; кроме того, $l\left(g_{i}\right)=\bar{l}\left(\bar{g}_{i}\right) \leqslant n$, так как $g_{i}-$ кратчайшие прообразы. Следовательно, $l\left(h_{i}\right) \leqslant 3 n$ для всех $i=1, \ldots, l$. По определению функции роста общего ранга с учетом оценки (4.7) получаем

$$
r_{H}(3 n) \geqslant l=c_{0} \gamma_{\bar{G}}(n)
$$

откуда и следует требуемое неравенство.

Доказательство теоремы 2.3. Напомним обозначения, введенные в теореме 2.3. Пусть $G=F / \gamma_{m} R \in \mathscr{K}, A=R / \gamma_{m} R, \varepsilon-$ естественный гомоморфизм $G \rightarrow \bar{G}=F / R, r_{\bar{H}}-$ функция роста обшего ранга подгруппы $\bar{H}=\varepsilon(H)$ в $\bar{G}$, $\gamma_{G / A H}-$ функция роста факторгрупшы $G / A H$.

Обозначим через $M=\varphi^{-1}(A H)$ полньй прообраз подгруппы $A H$ при естественном гомоморфизме $\varphi: F \rightarrow G$. Рассмотрим факторгруппу $F / \gamma_{m} M$. Так 
как $R \leqslant M$, то $\gamma_{m} M$ содержит $\gamma_{m} R$ и сушествует естественный гомоморфизм $\psi: G \rightarrow F / \gamma_{m} M$. Ясно, что образ $\psi(H)$ лежит в $B=M / \gamma_{m} M$. Кроме того, группа $\psi(H)$ нетривиальна. Действительно, пусть $\psi(H)=1$. Тогда $H \nless \&$, так как иначе $M=R$, гомоморфизм $\psi$ является изоморфизмом и $H=1$. Условие $\psi(H)=1$ равносильно $H \leqslant \gamma_{m}(A H)$, откуда, переходя к факторгруппе по $A$, получаем $A H / A \leqslant \gamma_{m}(A H / A)$, что невозможно в силу разрешимости (по определению класса $\mathscr{K})$ и нетривиальности групшы $A H / A$. Итак, $\psi(H) \neq 1$.

Возьмем такое число $l \geqslant 1$, что факторгруппа $\left(\psi(H) \gamma_{l} B\right) / \gamma_{l} B$ нетривиальна, но в тоже время $\left(\psi(H) \gamma_{l} B\right) / \gamma_{l-1} B=1$. Наконец, пусть $\widetilde{G}=F / \gamma_{l} M, \widetilde{H}$ - образ подгруппы $H$ при естественном гомоморфизме $\sigma: G \rightarrow \widetilde{G}$. Кроме того, обозначим $\widetilde{A}=M / \gamma_{l} M \cong B / \gamma_{l} B$. Очевидно, что группа $\widetilde{G}$ и подгруппы $\widetilde{H}$ и $\widetilde{A}$ удовлетворяют всем условиям леммы 4.7 , а значит,

$$
\gamma_{G / A H} \sim \gamma_{F / M} \preceq r_{\widetilde{H}}
$$

Учитывая неравенство (3.17), получаем

$$
\gamma_{G / A H} \preceq r_{H}
$$

Нижняя оценка в неравенстве (2.1) доказана.

Докажем верхнюю оценку. Для этого фиксируем какое-нибудь конечное порождаюшее множество $X$ группы $G$ и соответствуюшую функцию длины $l$. Через $\bar{l}$ обозначим функцию длины на $\bar{G}$ относительно $\varepsilon(X)$. Пусть

$$
\begin{aligned}
(A H)_{n} & =\operatorname{gp}\{h \in A H: l(h) \leqslant n\} \\
\bar{H}_{n} & =\operatorname{gp}\{\bar{h} \in \bar{H}: \bar{l}(\bar{h}) \leqslant n\} .
\end{aligned}
$$

Из результатов работы [1] (см. (1.2)) следует, что функция роста ранга полного прообраза $M=\varphi^{-1}(A H)$ эквивалентна $\gamma_{F / M}$, а значит, и $\gamma_{G / A H}$. В частности,

$$
\operatorname{rank}(A H)_{n}=r_{A H}^{*}(n) \leqslant r_{M}(n) \leqslant k_{1} \gamma_{G / A H}\left(c_{1} n\right)
$$

для некоторых $c_{1}, k_{1}=$ const.

Пусть $r_{\bar{H}}(n)=\operatorname{rank} \bar{K}=s$, где $\bar{H}_{n} \leqslant \bar{K}$ и $\bar{h}_{1}, \ldots, \bar{h}_{s}-$ минимальная система порождающих групшы $K$. Заметим, что по определению функции роста общего ранга $s$ не превосходит ранга $\bar{H}_{n}$, который, в свою очередь, не больше, чем $\operatorname{rank}(A H)_{n}$. Таким образом,

$$
s \leqslant k_{1} \gamma_{G / A H}\left(c_{1} n\right) .
$$

Зафикисируем какие-нибудь прообразы $h_{1}, \ldots, h_{s}$ элементов $\bar{h}_{1}, \ldots, \bar{h}_{s}$ в группе $H$ и положим

$$
P=\operatorname{gp}\left(\left\{h_{1}, \ldots, h_{s}\right\} \cup(A H)_{n}\right), \quad S=P \cap H, \quad T=P \cap A .
$$

Ясно, что $S$ и $T$ нормальны в $P$. Покажем, что $P=S T$. Действительно, пусть $x$ - произвольный элемент группы $P$. Рассмотрим образ $\varepsilon(x)$. Очевидно, что $\varepsilon(x) \in \bar{H}_{n} ;$ значит, элемент $\varepsilon(x)$ представляется в виде некоторого слова $w\left(\bar{h}_{i}\right)$ 
в порождающих $\bar{h}_{1}, \ldots, \bar{h}_{s}$. Положим $y=w\left(h_{i}\right)$, где $w\left(h_{i}\right)$ - слово, полученное из $w\left(\bar{h}_{i}\right)$ заменой $\bar{h}_{i}^{ \pm 1}$ на $h_{i}^{ \pm 1}, z=y^{-1} x$. Тогда $x=y z$, причем $y \in S, z \in T$.

Легко заметить, что $\operatorname{rank} P \leqslant s+\operatorname{rank}(A H)_{n}$, откуда с учетом (4.8) и (4.9) получаем

$$
\operatorname{rank} P \leqslant 2 k_{1} \gamma_{G / A H}\left(c_{1} n\right)
$$

Кроме того, $\operatorname{rank} P / T=s$ игруппа $T$ нильпотентна ступени $t \leqslant m-1$. По лемме 4.2

$$
\operatorname{rank} S \leqslant 2^{m} s \cdot(\operatorname{rank} P)^{m-1}+2^{m} \cdot(\operatorname{rank} P)^{2 m-3} .
$$

Учитывая неравенство (4.10) и вхождение $H_{n} \subseteq S$, окончательно получаем

$$
r_{H}(n) \leqslant \operatorname{rank} S \leqslant k_{2}\left(r_{\bar{H}}\left(c_{2} n\right)\left(\gamma_{G / A H}\left(c_{2} n\right)\right)^{m-1}+\left(\gamma_{G / A H}\left(c_{2} n\right)\right)^{2 m-3}\right)
$$

для некоторых констант $c_{2}, k_{2}$. Теорема 2.3 доказана.

Доказательство следствия 2.1. Хорошо известно (и легко доказьвается), что всякая подгруппа полициклической группы конечно порождена. Поэтому в данном случае для любой подгруппы $H \triangleleft G$ функция $r_{\bar{H}}$ ограничена и неравенство (2.1) при $m=2$ превращается в эквивалентность

$$
r_{H} \sim \gamma_{G / A H}
$$

В работе [10] показано, что полициклическая группа имеет либо полиномиальньй, либо экспоненциальньй рост. Остается заметить, что в случае, когда группа $G / A H$ имеет экспоненциальный рост, мы получаем (2.3); если же рост $G / A H$ полиномиален, то группа $G / A H$ содержит нильпотентную подгруппу конечного индекса (см. [10]) и ее функцию роста можно вычислить (с точностью до эквивалентности) по формуле Басса (см. [11]). В последнем случае мы получим в точности (2.2).

Конечно порожденные подгруппы. Доказательство следствия 2.2. Сделаем сначала одно замечание. Пусть для некоторой подгрупшы $H$ в конечно порожденной групп $G$ функция роста обшего ранга $r_{H}$ ограничена. Отсюда совсем не обязательно следует конечность ранга подгруппы $H$. Например, в случае локально циклической подгруппы $H$ бесконечного ранга получим

$$
r_{H} \equiv 1
$$

HO

$$
\operatorname{rank} H=\infty \text {. }
$$

В самом деле, ограниченность функции роста общего ранга подгруппы $H$ влечет ограниченность общего ранга в смысле Мальцева (см. [12]). Верно и обратное: если $H \subseteq G$ является группой конечного общего ранга $C$ в смысле Мальцева, то $r_{H}(n) \leqslant C$ для всех $n \in \mathbb{N}$.

Перейдем к доказательству следствия 2.2. Пусть факторгруппа $|G / A H|$ бесконечна. Тогда из нижней оценки в неравенстве (2.1) следует бесконечность функции роста общего ранга (а значит, и просто ранга) подгрупшы $H$. Если же $|G / A H|<\infty$, то как показывает сделанное выше замечание, из теоремы 2.3 следует лиш конечность общего ранга $H$ в смысле Мальцева. Однако, в этом случае нужный результат легко получается из леммы 4.2. В самом деле, из условия $|G / A H|<\infty$ следует конечность ранга подгруппы $A H$. В лемме 4.2 положим $S=H, T=A, P=A H$; тогда получим $\operatorname{rank} H<\infty$. 


\section{§5. Функции роста ранга и многообразия групп}

Лемма о длине элемента. В настоящем параграфе мы будем рассматривать групшы вида $G=F /[R, R] \in \mathscr{K}$. По лемме 4.3 всякая такая группа вложима в дискретное сплетение $S=U \operatorname{wr} \bar{G}$, где $U$ - свободная абелева группа подходяшего ранга, $\bar{G}=F / R$.

ОПРЕДЕЛЕНИЕ 5.1. Пусть $G$ - группа вида $F /[R, R]$. Запись образа элемента $g \in G$ при вложении $\theta$ группы $G$ в сплетение $S=U$ wr $\bar{G}$ вида

$$
\theta(g)=\bar{g} \cdot u_{i_{1}}^{\alpha_{1}}\left(h_{1}\right) \ldots u_{i_{l}}^{\alpha_{k}}\left(h_{l}\right)
$$

где $\alpha_{j} \neq 0, u_{i_{j}}\left(h_{j}\right)$ - свободные порождающие базы сплетения и $u_{i_{j}}\left(h_{j}\right) \neq u_{i_{k}}\left(h_{k}\right)$ при $j \neq k$, назовем канонической (такая запись единственна с точностью до перестановки элементов $u_{j}$, см. [8]). Размером канонической записи назовем число $p(g)$ различных элементов $u_{i_{j}}\left(h_{j}\right)$ в $(5.1)$.

Нетрудно установить, что $p(g)=\operatorname{card} \operatorname{supp} g$, так как в данном случае множество элементов $h_{j}$ в записи (5.1) совпадает с носителем элемента $g$.

ЛЕмма 5.1. Пусть $G$ - әруппа вида $F /[R, R], l$ - функиия длины на $G$ относительно порожсдающего множсества, полученного естественным образом из базиса группь $F$. Тогда для всякого әлемента $g \in G$ длина $l(g)$ не меньше размера канонической записи его образа, т.е.

$$
l(g) \geqslant p(g)
$$

ДокАЗАТЕЛЬСтво проведем индукцией по $l(g)$. При $l(g)=1$ утверждение следует из явного вида вложения $\theta$ (см. лемму 4.3). Пусть $l(g)=k>1$. Тогда $g=x^{ \pm 1} g_{0}$, где $l\left(g_{0}\right)=k-1, x-$ порождаюший элемент группы $G$. Рассмотрим для определенности случай $g=x g_{0}$. Положим $\theta\left(g_{0}\right)=\bar{g}_{0} t, \theta(x)=\bar{x} u(1)$, где $\bar{g}_{0}, \bar{x} \in \bar{G}, t \in T$, а $u(1)$ - один из элементов свободного порождающего множества подгруппы $U(1)$ в базе сплетения $T$. Тогда $\theta(g)=\bar{x} u(1) \cdot \bar{g}_{0} t=\bar{x} \bar{g}_{0} u\left(\bar{g}_{0}\right) t$. При этом

$$
p(g) \leqslant p\left(g_{0}\right)+1 \leqslant l\left(g_{0}\right)+1=l(g)
$$

Случай $g=x^{-1} g_{0}$ рассматривается аналогично. Лемма доказана.

Доказательство теоремы 2.4. Докажем сначала включение $\Omega\left(\mathfrak{S}_{2}\right) \subseteq \Omega_{p}$. В силу неравенства (3.17) достаточно доказать полиномиальность функций роста общего ранга подгрупп в свободных метабелевых группах. Итак, пусть $G-$ свободная метабелева группа конечного ранга, $H \leqslant G$. Фиксируем какую-нибудь свободную систему порождающих $X$ группы $G$ и соответствующую функцию длины $l$. Пусть, как обычно, $\bar{H}$ - образ подгруппы $H$ при естественном гомоморфизме $\varepsilon: G \rightarrow \bar{G}=F / F^{\prime}$. Без ограничения общности можно предположить, что $\bar{H}$ обладает таким базисом $\left\{\bar{h}_{1}, \ldots, \bar{h}_{k}\right\}$, что при всех $i$ имеет место $\bar{h}_{i}=\varepsilon\left(x_{i}^{d_{i}}\right)$ для некоторых $x_{i} \in X$ и $d_{i} \in \mathbb{N}$. 
Рассмотрим множество $Y$, построенное следуюшим образом. Во-первых, включим в $Y$ некоторые прообразы $h_{1}, \ldots, h_{k} \in H$ элементов $\bar{h}_{1}, \ldots, \bar{h}_{k}$. Далее, рассмотрим некоторый элемент $h \in H$ длины не более $n$. Элемент $\varepsilon(h)$ лежит в $\bar{H}$ и имеет длину относительно $\varepsilon(X)$ не более $n$; ясно, что $\varepsilon(h)$ представляется в виде слова $w_{h}\left(\bar{h}_{j}\right)$ в алфавите $\left\{\bar{h}_{1}^{ \pm 1}, \ldots, \bar{h}_{k}^{ \pm 1}\right\}$ длины не более $n$. Добавим к множеству $Y$ все элементы вида $h^{-1} w_{h}\left(h_{j}\right)$, где $w_{h}\left(h_{j}\right)$ - слово, полученное из $w_{h}\left(\bar{h}_{j}\right)$ заменой $\bar{h}_{j}$ на $h_{j}$ и $\bar{h}_{j}^{-1}$ на $h_{j}^{-1}$, а $h$ пробегает множество всех элементов подгруппы $H$, имеющих длину не более $n$. Ясно, что $h^{-1} w_{h}\left(h_{j}\right) \in G^{\prime}$ при любом $h$. Кроме того, при любом $h$ длины не более $n$

$$
l\left(h^{-1} w_{h}\left(h_{j}\right)\right) \leqslant c n
$$

для некоторой константы $c$. Легко проверить, что построенное множество $Y$ порождает некоторую подгруппу в $H$, содержащую $H_{n}$.

Заметим, что подгруппа $B$, порожденная элементами вида $h^{-1} w_{h}\left(h_{j}\right) \in Y$, лежит в $A_{c n}$. В силу теоремы 2.3 и полиномиальности функции роста абелевой факторгруппы $G / A$ имеем $\operatorname{rank} B \leqslant \operatorname{rank} A_{c n} \leqslant c_{1} n^{d}$ при некоторых $c_{1}, d=$ const. Таким образом,

$$
r_{H}(n) \leqslant \operatorname{rankgp} Y \leqslant k+\operatorname{rank} B \leqslant c_{2} n^{d}
$$

для некоторой константы $c_{2}$.

Теперь покажем, что всякая функция $f \in \Omega_{p}$ реализуется с точностью до эквивалентности в качестве функции роста обшего ранга некоторой подгруппы в свободной метабелевой группе. По определению полиномиальной функции найдутся такие константы $c$ и $s$ (которые можно считать натуральньми), что $f(n) \leqslant c n^{s}$ для всех $n \in \mathbb{N}$. Возьмем свободную метабелеву группу $G$ со свободным порождаюшим множеством $X=\left\{x_{1}, \ldots, x_{s+2}\right\}$ и рассмотрим множество элементов $W=\left\{w_{\lambda_{1}, \ldots, \lambda_{s}}\right\}, \lambda_{j} \in \mathbb{Z}$, вида

$$
w_{\lambda_{1}, \ldots, \lambda_{s}}=\left[x_{s+1}^{l}, x_{s+2}^{l}\right]^{x_{1}^{\lambda_{1}} \ldots x_{s}^{\lambda_{s}}}
$$

где $l=\left|\lambda_{1}\right|+\cdots+\left|\lambda_{s}\right|$.

Ясно, что носители различных элементов множества $W$ попарно не пересекаются. Обозначим $W_{n}=\left\{w \in W: l_{G}(w) \leqslant n\right\}$, где $l_{G}$ - функция длины на $G$ относительно $X$. Тогда для некоторой достаточно большой константы $k \in \mathbb{N}$ имеем card $W_{k n} \geqslant n^{s} \geqslant f(n)$. Значит, можно выбрать такое подмножество $V \subseteq W$, что

$$
\operatorname{card} V_{k n}=f(n)
$$

где $V_{n}=\left\{v \in V: l_{G}(v) \leqslant n\right\}$. Рассмотрим подгруппу $H=\operatorname{gp} V$ и покажем, что

$$
r_{H} \sim f
$$

Действительно, из построения множества $V$ следует, что $V_{k n} \subseteq H_{k n}$. По леммe 4.6

$$
r_{H}(k n) \geqslant \operatorname{card} V_{k n}=f(n) .
$$

Далее, пусть $h \in H, h=w_{1}^{\mu_{1}} \ldots w_{k}^{\mu_{k}}$, где $w_{1}, \ldots, w_{k}$ - различные элементы множества $W, \mu_{1} \neq 0, \ldots, \mu_{k} \neq 0$ и длина $l_{G}(h) \leqslant n$. Заметим, что если носители 
элементов $g_{1}, g_{2} \in[G, G]$ не пересекаются, то supp $g_{1} g_{2}=\operatorname{supp} g_{1} \cup \operatorname{supp} g_{2}$. Поэтому, учитьвая лемму 5.1 , имеем $l_{G}(h) \geqslant p(h)=p\left(w_{1}^{\mu_{1}} \ldots w_{k}^{\mu_{k}}\right) \geqslant p\left(w_{j}\right)$ для любого $j$. Непосредственное вычисление показывает, что каноническая запись элемента $\theta\left(\left[x_{s+1}^{l}, x_{s+2}^{l}\right]\right)$ имеет вид

$$
\begin{aligned}
& u_{s+1}^{-1}(1) u_{s+1}^{-1}\left(\bar{x}_{s+1}\right) \ldots u_{s+1}^{-1}\left(\bar{x}_{s+1}^{l-1}\right) u_{s+2}^{-1}\left(\bar{x}_{s+1}^{l}\right) u_{s+2}^{-1}\left(\bar{x}_{s+1}^{l} \bar{x}_{s+2}\right) \ldots \\
& \ldots u_{s+2}^{-1}\left(\bar{x}_{s+1}^{l} \bar{x}_{s+2}^{l-1}\right) u_{s+1}\left(\bar{x}_{s+2}^{l} \bar{x}_{s+1}^{l-1}\right) \ldots \\
& \quad \ldots u_{s+1}\left(\bar{x}_{s+2}^{l} \bar{x}_{s+1}\right) u_{s+1}\left(\bar{x}_{s+2}^{l}\right) u_{s+2}\left(\bar{x}_{s+2}^{l-1}\right) \ldots u_{s+2}\left(\bar{x}_{s+2}^{1}\right) u_{s+2}(1) .
\end{aligned}
$$

Отсюда следует, что для всякого элемента $w_{j}=w_{\lambda_{1}, \ldots, \lambda_{s}}$

$$
p\left(w_{j}\right)=4 l \geqslant \frac{2}{3} l_{G}\left(w_{j}\right)
$$

так как $l_{G}\left(w_{j}\right) \leqslant 6 l$. Поэтому для всех $j=1, \ldots, k$ имеем $l_{G}\left(w_{j}\right) \leqslant \frac{3}{2} l_{G}(h) \leqslant \frac{3}{2} n$. Значит, $H_{n} \subseteq V_{\left[\frac{3}{2} n\right]}$ и по определению функции роста общего ранга

$$
r_{H}(n) \leqslant f\left(\left[\frac{3}{2} n\right]\right) \leqslant f(2 n)
$$

Комбинируя неравенства (5.4) и (5.6), получаем (5.3). Теорема 2.4 доказана.

Доказательство теоремы 2.5 по сути повторяет доказательство теоремы 2.4. Пусть $G$ - свободная разрешимая группа ступени 3 , свободно порожденная элементами $x_{1}, x_{2}, x_{3}, x_{4}, x_{5}, x_{6}$. В качестве $W$ следует выбрать подмножество таких элементов $w_{\bar{g}} \in G$, индексированных элементами $\bar{g} \in G_{0} / G_{0}^{\prime \prime}$, где $G_{0}=\operatorname{gp}\left\{x_{1}, x_{2}\right\}$, что

$$
w_{\bar{g}}=\left[\left[x_{3}^{l}, x_{4}^{l}\right],\left[x_{5}^{l}, x_{6}^{l}\right]\right]^{g},
$$

где $g$-кратчайший (относительно $x_{1}, x_{2}$ ) представитель элемента $\bar{g}$ в $G_{0}$ (ясно, что $w_{\bar{g}}$ не зависит от выбора этого представителя), a $l$ - длина элемента $g$ относительно $x_{1}, x_{2}$. Ввиду экспоненциальности роста свободной метабелевой группы $G_{0} / G_{0}^{\prime \prime}$ множество $W$ имеет экспоненциальный рост.

Так же, как и выше, для заданной функции $f$ не более чем экспоненциального роста выбирается подмножество $V \subseteq W$ со свойством

$$
\operatorname{card} V_{k n}=f(n)
$$

Ясно, что supp $w_{\bar{g}_{1}} \cap \operatorname{supp} w_{\bar{g}_{2}}=\varnothing$ при $\bar{g}_{1} \neq \bar{g}_{2}$. Как следствие получаем нижнюю оценку функции роста обшего ранга подгрупшы $H=\operatorname{gp} V$.

Находя в явном виде каноническую запись образа элемента $\left[\left[x_{3}^{l}, x_{4}^{l}\right],\left[x_{5}^{l}, x_{6}^{l}\right]\right]$, убедимся, что вместо неравенства (5.5) имеет место

$$
p\left(w_{\bar{g}}\right)=16 l \geqslant \frac{8}{9} l_{G}\left(w_{\bar{g}}\right)
$$

где $l_{G}$ - функция длины на $G$ относительно $\left\{x_{1}, \ldots, x_{6}\right\}$. Рассуждая далее так же, как и в доказательстве теоремы 2.4 , получаем верхнюю оценку для функции роста общего ранга подгруппы $H$. 


\section{Список литературы}

1. Osin D. V. On the generating set's growth functions for subgroups of free groups // Internat. J. Algebra Comput. 1999. V. 9. № 1. P. 41-50.

2. Rosenmann A. When Schreier transversals grow wild // Preprint, 1997.

3. Howson A. G. On the intersection of the finitely generated free groups // J. London Math. Soc. 1954. V. 29. P. 428-434.

4. Эйделькинд Д. И. О конечно порожденных нормальных делителях полинильпотентных групп // Сиб. матем. журн. 1968. Т. 9. №1. С. 236-239.

5. Baumslag G. Some theorems on the free groups of certain product varieties // J. Combin. Theory. 1967. V. 2. P. 77-99.

6. Ol'shanskii A. Yu. Distortion functions for subgroups // Geometric group theory down under (Canberra, Australia, 1996). Walter de Gruyter, 1999. P. 281-291.

7. Холл Ф. Нильпотентные группы // Математика. Сб. пер. 1968. Т. 12. № 1. С. 3-36.

8. Нейман Х. Многообразия групп. М.: Мир, 1969.

9. Шмелькин А. Л. Сплетения и многообразия групп // Изв. АН СССР. Сер. матем. 1965. T. 29. № 1. C. 149-170.

10. Wolf J. A. Growth of finitely generated solvable groups and curvature of Riemannian manifolds // J. Differential Geom. 1968. V. 2. P. 421-446.

11. Bass $H$. The degree of polynomial growth of finitely generated nilpotent groups // Proc. London Math. Soc. (3). 1972. V. 25. P. 603-614.

12. Мальцев А. И. О группах конечного ранга // Матем. сб. 1948. Т. 22. № 2. С. 351-352.

Московский государственнњй

университет им. М.В. Ломоносова
Поступила в редакцию

21.10.1998 и 13.11 .1998 